\title{
Perspective of Radiology in the next one hundred years
}

\author{
Claudia da Costa Leite ${ }^{1}$, Edson Amaro Júnior ${ }^{2}$, Carlos Alberto Buchpiguel ${ }^{3}$, Giovanni Guido Cerri ${ }^{4}$
}

$\mathrm{T}^{1}$ he history of Medicine is parallel to the history of civilizations, there are Medical reports from the ancients (Egyptians, Babylons and Greeks). Even though the modern Medicine has evolved over the last century, it has a long tradition. On the other hand, the history of Radiology began in the XIX century, when Wilhewn Conrad Röentgen in November $8^{\text {th }}, 1895$ discovered the X-ray and later was able to make the first images from his wife hand. This was the begging of looking inside the patient in a novel way ${ }^{1}$.

From Röentgen to the present time the Radiology, now better described as Diagnostic Imaging has revolutionized the modern medicine. Radiology evolved from the $\mathrm{X}$-ray, to fluoroscopy very fast. Other modalities, such as angiography, were introduced after the development of intravenous iodinated contrast. The first angiocardiography was done by Forsmann in 1929 after a successful attempt of cardiac catetherization ${ }^{2}$. The ultrassonography was introduced to medical imaging after the development of B-mode and in 1958 the first images of abdominal disease were presented ${ }^{3}$. In 1970, Godfrey Hounsfield together with Dr. Jamie Ambrose, showed the first computed tomography (CT) images of the brain changing definitively the radiology from merely projection images to sectional images. The development of magnetic resonance imaging (MRI) through the work of Edward Mills Purcell, Paul C. Lauterbur, Raymond V. Damadian and Sir Peter Mansfield allowed that in 1977 the first human scan was done in a MRI prototype after 5 hours of examination ${ }^{4}$. From there to now the technological advances have progressed very rapidly.

The Nuclear Medicine was launched by the development of radioactive tracers and their scintillation detection methods. In the beginning radioactive iodine was used to treat thyroid cancer. From the Geiger count (1928) to Anger (1957) that constructed the prototype of the modern gamma camera, to the longitudinal tomographic scanner ${ }^{5}$ and transaxial tomography (Harper 1965) to the development of SPECT (single photon emission computed tomography by Jaszczak 1979) and PET (positron emission tomography by Michael Phelps and Edward Hoffman in 1975) there was an enormous development of radiotracers as well as of equipments that allow the achievement of functional images with radiotracers.

Parallel to the development of Radiology and

1. Professora Associada do Departamento de Radiologia e Oncologia da Faculdade de Medicina da Universidade de São Paulo. Chefe do Ensino e Pesquisa do Instituto de Radiologia do Hospital das Clínicas da FMUSP. Associated Professor, Department of Radiology, University of North Carolina at Chapel Hill.

2. Professor Doutor MS-5 do Departamento de Radiologia da Faculdade de Medicina da Universidade de São Paulo.

3. Professor Titular do Departamento de Radiologia e Oncologia da Faculdade de Medicina da Universidade de São Paulo. Diretor do Serviço de Medicina Nuclear e Imagem Molecular do Instituto de Radiologia do Hospital das Clínicas da Faculdade de Medicina da Universidade de São Paulo.

4. Professor Titular do Departamento de Radiologia e Oncologia da Faculdade de Medicina da Universidade de São Paulo. Mailling Address: Claudia da Costa Leite. Instituto de Radiologia. Rua Dr. Enéas de Carvalho Aguiar, 251. Pinheiros. 05403-000 São Paulo, SP, Brasil. 
Nuclear Medicine there was the need of a documentation of these medical images. The development of radiographic films was great in the last century, but in the last years, the films were replaced by the computational midia as the RIS/ PACS (Radiological Information Systems/Picture Archive Computer System) was introduced and the filmless concept was widely accepted.

The history of the Department of Radiology of the University of São Paulo began in the Santa Casa de Misericórdia under the supervision of Dr. Rafael de Barros. In that time the radiology contents were presented in the Applied Physics and Biology discipline. In 1944 the Radiology section was transferred to the new Hospital das Clínicas, and the radiology residency was opened. Radiology as a department was official as it is only in 1968. As the technology advanced the Department of Radiology has also changed a lot, from the conventional radiograph to ultrasonography, computed tomography, magnetic resonance imaging, nuclear medicine and interventional radiology. The first computed tomography of the Hospital das Clínicas began its operation in 1986, while the first MR equipment in a Brazilian public hospital was installed in this hospital in 1992. The Instituto de Radiologia (InRad) was created in 1994, allowing a more rapid development of the Radiology Department and its consolidation as an important Brazilian radiology educational center ${ }^{6}$. The changes in the manner as the radiologist interacts with its professional has also changed a lot, the first group of emergency radiology was introduced in 1998 followed by the increase of the procedures guided by radiology, named as non-vascular interventional radiology. The radiology evolved from a diagnostic to a diagnostic/therapeutic role. Another important development was the acquisition of the RIS/PACS and the transformation to a filmless Radiology Department that happened in 2007.

During those years the Radiology has increased its concern about safety and efficacy. Each time there was a more careful overview of the radiation dose exposure. On the other hand, the images have more and more resolution and their acquisition is faster and faster. The diagnostic imaging has moved from a structural analysis to a quantitative and functional imaging and will move in the next years to the molecular imaging, the associating structural, functional and the molecular approach.
For the next years many changes are expected: precision radiology, minimally invasive radiology treatments, molecular imaging, automatic pattern recognition, quantitative imaging, among others.

The actual trend of radiology is to migrate towards a more personalized way to detect abnormalities and diseases (precise medicine), linking diagnosis and therapy as a single information to allowing a better clinical outcome. New diagnostic and therapeutic strategies tailored for each patient requirements are necessary ${ }^{7}$. Radiology has changed in the last decades introducing a new therapeutic approach that evolved a lot with the introduction of minimally invasive procedures. In the future Radiology will have to take the individual variability into account in opposition to an indiscriminate medical approach that do not respect the different genotypes and clinical characteristics, it will be a precision radiology approach.

The future of diagnostic imaging, including nuclear medicine and radiology is to deeply enter in the field of molecular biology, where the detection of different diseases are now based on their molecular profile more than on their anatomic or physiological consequences. As molecular medicine develops and gain more clinical importance and value in the routine clinical workup, wider range of radiopharmaceuticals will be developed to tracer different biological processes inside the patient's body. The concept of theranostics (diagnosis to better apply therapy) will be the future of molecular imaging, and consequently nuclear medicine and radiology. Besides that advance in biology, more sophisticated hybrid systems are being introduced to link anatomical and molecular information together. The examples of hybrid systems in the present era are PET-CT (positron emission tomography and computed tomography coupled) and PET-MRI (positron emission tomography and magnetic resonance imaging coupled) that are already a reality.

Another important expected development is the automation based on visual pattern recognition as well as quantitative imaging in which quantifiable features/ biomarkers are extracted from images for the evaluation of normal or the severity, degree of change in an abnormal condition that are assessed generating a more objective approach of imaging findings ${ }^{8,9}$.

In future years to come radiologist are likely to 
act more as consultants and share responsibilities in patient management. Evidence from medical practice including trends to integrate medical diagnose, practice and treatment to each patient routine should thrive only if market practices allow for incorporation of recent innovation trends. In fact, nowadays the current thinking points to de-hospitalization, wearables (with so called "internet of things") and other empowering tools that allows for each person a better knowledge of his/her health status. Radiologist comes directly into this new agenda as "gate-keepers" for medical interactions. It is expected that techniques will bring together information from anatomy, physiology and "omics" (suffix related to genomics, proteomics, metabolomics, interactomics, socionomics and such). In this scenario, what we currently have as parts of the 'patient enigma' should be integrated in an imaging background. "Practice prophets" propose that, instead of sending a patient to have blood (and other fluids/ tissues) samples analyzed in a pathology lab, the patient will have imaging examination (usually in more than one modality) and then perform biopsies, all integrated in only one imaging procedure. Imagine having immunochemical essays performed inside patient's body and pathology assessment based in either non-invasive imaging or (as above mentioned) obtained with minimal harm - and at the same time, delivering the treatment. All those points will require a more pro-active role for the radiologist. The imaging diagnostic specialty should mold itself in an imaging diagnostic/prognostic/treatment procedure - and all this in close relationship with the carrying physician.

\section{REFERENCES}

1. European Society of Radiology (ESR). The story of radiology. Vienna, Austria: European Society of Radiology; 2012. v.1. Available from: http://www.internationaldayofradiology. com/wp_live_idor_uai3A/wp-content/uploads/2012/10/ IDOR_2012_Story-of-Radiology_RZ_lowres.pdf.

2. Wilms G, Baert AL. The history of angiography. J Belge Radiol. 1995;78(5):299-302.

3. Christopher L, Moore MD, Joshua A, Copel MD. Point-ofcare ultrasonography. N Engl J Med. 2011;364:749-57. doi: 10.1056/NEJMra0909487.

4. Tesla Memorial Society of New York. A short history of the magnetic resonance imaging (MRI). Available from: www. teslasociety.com/mri.htm.

5. Carlson S. A Glance at the history of nuclear medicine. Acta Oncol, 1995;34:1095-102. doi: 10.3109/02841869509127236.

6. Belarmino A. Departamento de Radiologia. In: Mota A, Marinho MGSMC, organizadores. Trajetória da Faculdade de
In other words: radiologists are thought to have a major role in radiology practice, especially when patients are in the hospitals. Clinical assessments and minor interventions are likely to happen in patient's home or in centers located in small communities, under medical guidance. Imaging suites acting as the 'stage action' for such procedures will demand a completely different training and orchestration of learning methods in radiology.

In more wide aspects, one can imagine a scenario 100 years from now in which all of us should have a "health tracker and would be able to take decisions about our health status on a sole base of feedbacks from medical systems. Sensing or probing our body should be a matter of continuous non-invasive sampling at molecular level. No need to set appointments and go to a hospital/clinic to have your body scanned: this could be done at home and even perhaps by instruments invisible to us. Most of current human role from radiologist today is in interfacing with technology and translating the "data message to other physician, and the physician to the patient. In the future, this communication with not even be conscious: autonomic feedback and brain-machine interfaces might dramatically shorten the gap. If this "sci-fi" view comes to reality, a complex change of human interactions might take place. The role of a medical doctor (not only a radiologist) will be quite challenging, as the "intangible items" of medical practice governing human interactions will turn into the critical ones. Our relationships and trust, care and human "biological communication skills will be key ${ }^{10}$.

Medicina da Universidade de São Paulo - aspectos históricos da Casa de Arnaldo (departamentos da Faculdade de Medicina da Universidade de São Paulo). São Paulo: CD.G Casa de Soluções e Editora; 2012. v.2, p.264-83.

7. Mirnezami R, Nicholson J, Darzi, A. Preparing for precision medicine. N Engl J Med. 2012;366(6):489-91. doi: 10.1056/ NEJMp1114866.

8. Buckler AJ, Bresolin L, Dunnick NR, Sullivan DC, Aerts HJ, Bendriem B, et al. Quantitative Imaging Test Approval and Biomarker Qualification: interrelated but distinct activities. Radiology. 2011;259(3): 875-84. doi: 10.1148/ radiol.10100800.

9. Buckler AJ, Bresolin L, Dunnick NR, Sullivan DC; Group. A Collaborative Enterprise for Multi-stakeholder Participation in the Advancement of Quantitative Imaging. Radiology. 2011;258(3):906-14. doi: 10.1148/radiol.10100799.

10. Krestin GP. Maintaining identity in a changing environment: the professional and organizational future of radiology. Radiology. 2009;250(3):612-7. doi: 10.1148/radiol.2503081791. 